\title{
Congenital diaphragmatic hernia: association between pulmonary vascular resistance and plasma thromboxane concentrations
}

\author{
W D A FORD, M J JAMES, AND J A WALSH \\ Department of Paediatric Surgery, Adelaide Children's Hospital and Department of Surgery, Flinders \\ Medical Centre, Bedford Park, South Australia
}

SUMmaRY After repair of a Bochdalek diaphragmatic hernia, shunting of blood away from the lungs produced progressive hypoxaemia in a neonate. Changes in plasma concentrations of the vasoconstrictor prostanoid thromboxane were associated with changes in pulmonary vascular resistance in this infant. Plasma concentrations of the vasodilator prostanoid prostacyclin were also monitored during acute changes in pulmonary vascular resistance. Tolazoline administration was followed by reductions in pulmonary vascular resistance and in plasma thromboxane and prostacyclin values. Further studies indicated that tolazoline inhibits platelet thromboxane synthesis and vascular prostacyclin synthesis. These data suggest that thromboxane plays a part in the increased pulmonary vascular resistance that occurs after repair of congenital diaphragmatic hernia and that tolazoline may reverse pulmonary hypertension by a mechanism additional to its known action as an alpha adrenergic blocking agent and its effect on histamine release.

Pulmonary vasoconstriction is now recognised as a major contributing factor in the hypoxaemia and death that frequently follow a brief 'honeymoon' phase after repair of congenital diaphragmatic hernias. ${ }^{1}$ Blood gas exchange is adequate during this first phase, showing that simple pulmonary hypoplasia is not the only causative factor. ${ }^{1}$ Subsequently, however, gas exchange deteriorates as pulmonary vascular resistance increases and blood shunts away from the lungs through the ductus arteriosus or the foramen ovale.

Because animal studies have suggested that prostaglandins may have a role in the conversion of the fetal circulation to one where blood is oxygenated in the lungs, ${ }^{2}$ aberrant prostanoid metabolism may play a part in the pathological reversal to a fetal type circulation 12 to 48 hours after the surgical repair of congenital diaphragmatic hernia in the newborn. To test this hypothesis we monitored the plasma values of the stable degradation products of prostacyclin (a vasodilator prostaglandin) and thromboxane $\mathrm{A}_{2}$ (a vasoconstrictor prostanoid) in a critically ill neonate suffering from the effects of such a hernia.

\section{Methods}

Patient. A critically ill neonate (birthweight, $3.5 \mathrm{~kg}$ ) with a Bochdalek diaphragmatic hernia underwent successful hernia repair by a transabdominal approach. After surgery the infant was ventilated $\left(\mathrm{F}_{1} \mathrm{O}_{2}, 1.0\right)$ and blood was sampled at intervals simultaneously from indwelling cannulae in the right radial artery (pre-ductal blood) and the umbilical artery (post-ductal blood). The blood was used for acid base and blood gas analyses and prostaglandin estimations.

Plasma prostaglandin values. Blood was collected in heparinized tubes containing $30 \mu \mathrm{g}$ aspirin, and plasma was prepared by centrifugation. The amounts of prostacyclin and thromboxane $A_{2}$ were estimated by measuring the amounts of their stable degradation products, 6-keto $\mathrm{PGF}_{1 \alpha}$ and thromboxane $B_{2}$, respectively. Prostaglandin concentrations were measured in the lipid extracts of plasma (directly for thromboxane $\mathrm{B}_{2}$ and after thin layer chromatography for 6-keto $\mathrm{PGF}_{1 \alpha}$ ) by radioimmunoassays (RIA) which used commercially available antisera (Seragen Inc, USA). Corrections were made for extraction efficiencies and volumes of plasma extracted.

Prostaglandin synthesis-in vitro systems. Thromboxane $B_{2}$ synthesis was initiated in $0.1 \mathrm{ml}$ platelet 
rich plasma by the addition of thrombin (1U). The amount of thromboxane $B_{2}$ synthesised after $3^{\prime}$ at $37^{\circ}$ was measured by RIA of diluted aliquots $(20 \mu \mathrm{l})$ of platelet rich plasma. Slices $(1 \mathrm{~mm})$ of fresh umbilical artery synthesised 6-keto $\mathrm{PGF}_{1 \alpha}$ were incubated at $37^{\circ}$ for $10^{\prime}$ in $0.5 \mathrm{ml}$ Tyrode's buffer containing glucose $(1 \mathrm{mg} / \mathrm{ml})$. The amount of 6-keto $\mathrm{PGF}_{1 \alpha}$ synthesised was measured by RIA of lipid extracts. Corrections were made for extraction efficiency.

Tolazoline was added to these incubation systems at various concentrations. To measure the effect of tolazoline on 6-keto $\mathrm{PGF}_{1 \alpha}$ synthesis, umbilical artery slices were also preincubated with tolazoline for $5^{\prime}$ at $25^{\circ}$ before transfer to the final incubation system containing the same concentrations of tolazoline.

\section{Results}

At 1 hour and 5 hours after surgery, the $\mathrm{PaO}_{2}$ was $230 \mathrm{mmHg}$ in the right radial artery and $100 \mathrm{mmHg}$ in the abdominal aorta $\left(\mathrm{F}_{\mathrm{I}} \mathrm{O}_{2}, 1 \cdot 0\right)$. This difference in oxygenation (Fig. 1) indicated right to left shunting of blood through the ductus arteriosus or the foramen ovale, or both. This shunt was probably maintained by high pulmonary vascular resistance. High pulmonary vascular resistance was also suggested by an alveolar-aterial difference in oxygen tension $\left(\mathrm{A}-\mathrm{aDO}_{2}\right)$ of approximately $550 \mathrm{mmHg}$. During the period up to 17 hours after surgery, oxygenation increased in the umbilical artery blood samples (Fig. 1), a change that indicated decreased right to left shunting and was probably caused by decreased pulmonary vascular resistance. At 22 hours after surgery, $\mathrm{PaO}_{2}$ values had fallen to 25 $\mathrm{mmHg}$, indicating increased pulmonary vascular resistance. Tolazoline infusion $(1 \mathrm{mg} / \mathrm{kg} / \mathrm{hour})$ was begun 27 hours after surgery (Fig. 1). Oxygentaion improved and there was no evidence of right to left shunting 56 hours after surgery. The $\mathrm{F}_{\mathrm{I}} \mathrm{O}_{2}$ subsequently decreased over several days and ventilation was finally stopped, with no adverse clinical reactions.

Fig. 1 (a) shows that plasma thromboxane values were high during periods of apparent pulmonary hypertension ( 1 to 5 and 22 to 29 hours after surgery) and low during periods of apparent decreased pulmonary vascular resistance (11 to 17 hours after surgery and 33 hours after surgery to recovery). Furthermore, plasma thromboxane values decreased after tolazoline administration (Fig. 1 (a)).

Fig. 1 (b) shows that prostacyclin values in the right radial artery and umbilical artery samples varied independently until the right to left shunt
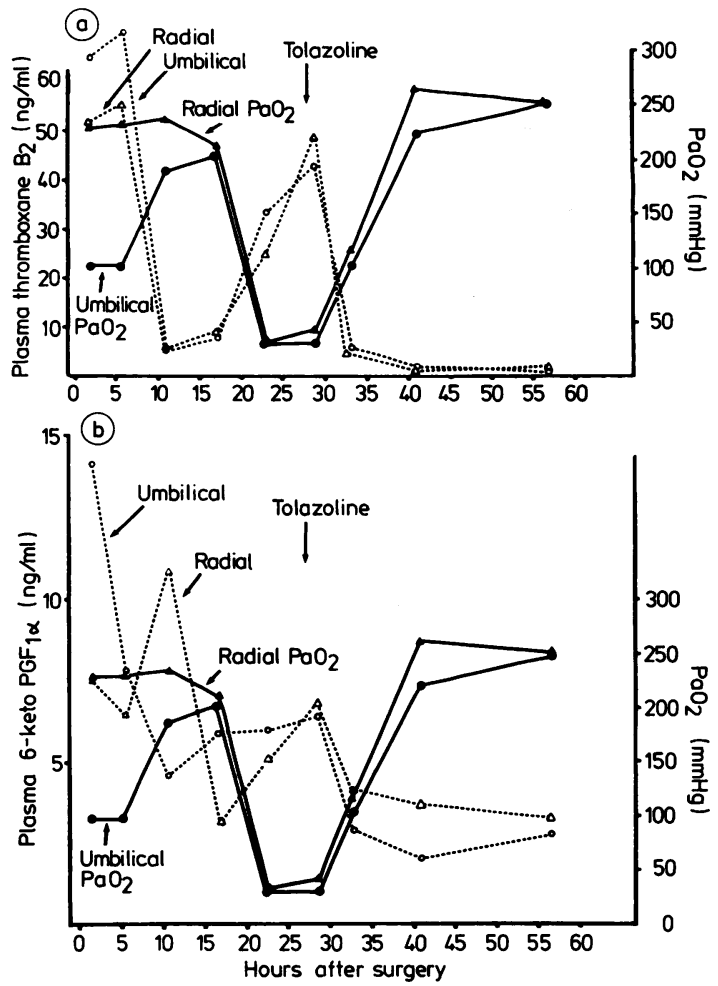

Fig. 1 Plasma prostanoid values and plasma $\mathrm{Pao}_{2}$ values after diaphragmatic hernia repair in a neonate. (a) Plasma thromboxane $B_{2}$ values (broken lines). (b) Plasma 6-keto $P G F_{l a}$ values (broken lines). Arterial $\mathrm{PaO}_{2}$ values (solid lines) are represented in (a) and (b). All values represent the results of single determinations.

stopped, after which time the values in each artery sample varied in a similar manner. The prostacyclin concentrations in the post-ductal (umbilical) blood sample decreased as the right to left shunt decreased and the prostacyclin values in the radial artery sample decreased before the increase in pulmonary vascular resistance 17 hours after surgery (Fig. 1 (b)). Plasma prostacyclin values also decreased after tolazoline administration (Fig. 1 (b)).

When the effects of tolazoline on vascular prostacyclin synthesis and platelet thromboxane synthesis were investigated in vitro, tolazoline inhibited the synthesis of both prostanoids (Fig. 2). Platelet thromboxane synthesis is inhibited at a lower concentration of tolazoline than that required to inhibit prostacyclin synthesis (Fig. 2).

\section{Discussion}

Fifty to $80 \%$ of neonates who present with a diaphragmatic hernia in the first 24 hours of age 

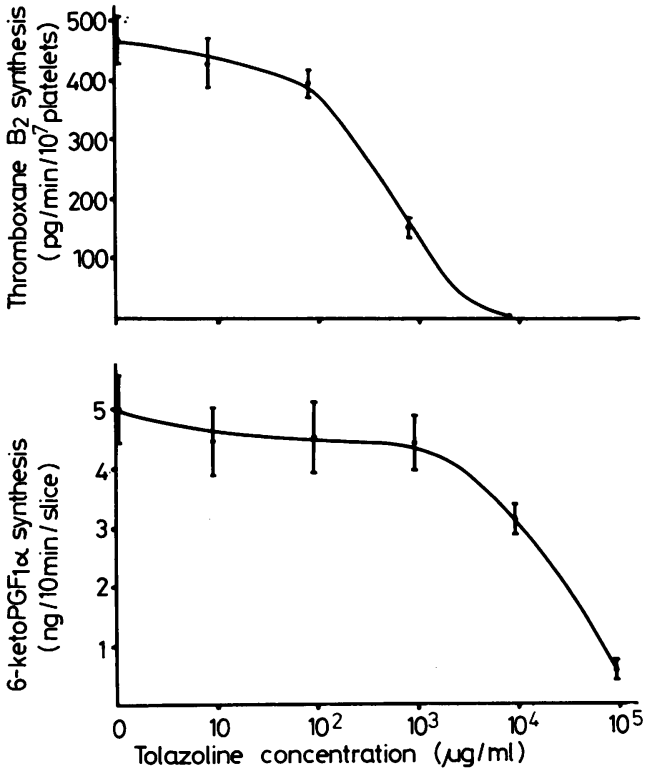

Fig. 2 The effect of tolazoline on (a) platelet thromboxane $B_{2}$ synthesis, and (b) vascular 6-keto $P G F_{1 \alpha}$ synthesis in vitro. The values represent the means (SEM) of triplicate determinations.

die. ${ }^{13}$ Initial mortality is the result of pulmonary hypoplasia. Many survive the immediate neonatal and preoperative periods, however, only to die as the result of increasing pulmonary vascular resistance and the shunting of blood away from the lungs. ${ }^{1}$ The immediate cause of the raised vascular resistance is unknown, but histological studies on the pulmonary vascular bed indicate an increased muscle mass in the prealveolar arterioles. ${ }^{1}$ Furthermore, these vessels are extraordinarily sensitive to the effects of changes in alveolar oxygen concentrations, $\mathrm{pH}$, blood oxygen values, and hormone values. ${ }^{1}$

Our study shows an association between plasma values of the vasoconstrictor prostanoid thromboxane $A_{2}$ and the apparent tone of the pulmonary vessels during the dramatic changes in pulmonary vascular resistance that occurred after diaphragmatic hernia repair in a neonate. The major site of thromboxane production in this instance is unknown, but the lungs are a potential site for the synthesis of the large amounts of thromboxane that we detected in plasma, because it has been shown that human lung parenchyma is capable of synthesising thromboxane. ${ }^{4}$ If the lungs are the major site of thromboxane production in this instance, the similar values of plasma thromboxane $B_{2}$ that we measured in radial and umbilical artery samples during the right to left shunting of blood, suggest that thromboxane $B_{2}$ has a plasma half life sufficient to allow equilibration within the total vascular space. A similar association between plasma thromboxane $\mathbf{B}_{2}$ values and pulmonary vascular resistance has been shown in sheep after infusion of complement activated plasma.

Plasma prostacyclin concentrations in the radial and umbilical artery samples varied independently during the right to left shunting of blood. This pattern suggests that values in the umbilical artery sample reflect prostacyclin synthesis by the ductus arteriosus, and values in the radial artery sample reflect synthesis by the lung - other explanations are, however, possible because many blood vessels are capable of synthesising prostacyclin. Nevertheless, it has been shown that lamb ductus arteriosus and human lung parenchyma can synthesise prostacyclin. ${ }^{46}$ Prostacyclin is a potent vasodilator and the data represented in Fig. 1 (b) are consistent with the hypothesis that prostacyclin production by the ductus arteriosus maintains its patency. As prostacyclin concentrations in the umbilical artery samples decreased, the right to left shunt stoppedprobably as a result of decreased pulmonary vascular resistance, or constriction of the ductus arteriosus, or both. Furthermore, the values of prostacyclin in the right radial artery samples decreased in the period before the pulmonary vessels constricted (11 to 17 hours after surgery), consistent with the hypothesis that prostacyclin production by the lungs acts as a pulmonary vasodilator in neonates.

These results indicate associations only between plasma values of prostanoids and the tone of the pulmonary vasculature in our patient, and do not allow the assignment of effector roles to these prostanoids. Nevertheless, these data suggest that acute pulmonary vasoconstriction in the neonate may be a consequence of the presence of a vasoconstrictor prostanoid (thromboxane $\mathrm{A}_{2}$ ) and possibly the absence of a vasodilator prostanoid (prostacyclin).

Tolazoline may cause pulmonary vasodilation by alpha blockade of sympathetic activity, but the evidence that pulmonary vascular tone is controlled by sympathetic activity is equivocal. ${ }^{7}$ Results from the in vivo and in vitro studies reported here suggest that tolazoline may cause vasodilatation by inhibiting thromboxane synthesis in addition to, or instead of, blocking alpha adrenergic activity.

It is noteworthy that tolazoline inhibits both thromboxane and prostacyclin synthesis, but with different dose/response curves. This potential for differential inhibition of prostanoid synthesis suggests that tolazoline may produce different cardiovascular effects at different doses. 
We thank $L$ Thorpe and $R$ Underwood for expert technical assistance and the nursing staff of the labour and delivery Ward at Flinders Medical Centre for their cooperation in obtaining fresh umbilical material.

\section{References}

1 Shochat SJ, Naeye RL, Ford WDA, Whitman V, Maisels MJ. Congenital diaphragmatic hernia. New concepts in management. Ann Surg 1979;190:332-41.

${ }^{2}$ Cassin S. Role of prostaglandins and thromboxanes in the control of the pulmonary circulation in the fetus and newborn. Semin Perinatol 1980:4:101-7.

${ }^{3}$ Harrison MR, Ross NA, de Lorimier AA. Correction of congenital diaphragmatic hernia in utero. III. Development of a successful surgical technique using abdominoplasty to avoid compromise of umbilical blood flow. $J$ Pediatr Surg 1981;16:934-42.
${ }^{4}$ Schulman ES, Newball HH, Demers LM, Fitzpatrick FA, Adkinson NF, Jr. Anaphylactic release of thromboxane $A_{2}$, prostaglandin $\mathrm{D}_{2}$, and prostacyclin from human lung parenchyma. Am Rev Respir Dis 1981;124:402-6.

5 Cooper JD, McDonald JWD, Ali M, Menkes E, Masterson J. Klement P. Prostaglandin production associated with the pulmonary vascular response to complement activation. Surgery 1980;88:215-21.

${ }^{6}$ Pace-Asciak CR, Rangaraj G. The 6-keto prostaglandin $F_{1 \alpha}$ pathway in the lamb ductus arteriosus. Biochim Biophys Acto 1977;486:583-5.

${ }^{7}$ Rudolph AM. Fetal and neonatal pulmonary circulation. Ann Rev Physiol 1979;41:383-95.

Correspondence to $\mathrm{Dr}$ M J James, Department of Surgery, Flinders Medical Centre, Bedford Park, South Australia 5042.

Received 26 October 1983 